\title{
Environmental chamber with controlled temperature and relative humidity for ice crystallization kinetic measurements by atomic force microscopy
}

Cite as: Rev. Sci. Instrum. 91, 023704 (2020); https://doi.org/10.1063/1.5132537

Submitted: 17 October 2019 . Accepted: 17 January 2020 . Published Online: 06 February 2020

Melisa M. Gianetti (D), Julián Gelman Constantin (D), Horacio R. Corti (D), and M. Paula Longinotti (D)

\section{ARTICLES YOU MAY BE INTERESTED IN}

Development of dual bias modulation electrostatic force microscopy for variable frequency measurements of capacitance

Review of Scientific Instruments 91, 023702 (2020); https://doi.org/10.1063/1.5127219

Achievement of high power and long pulse negative ion beam acceleration for JT-60SA NBI Review of Scientific Instruments 91, 023506 (2020); https://doi.org/10.1063/1.5131302

Design, construction, and operation of an $18 \mathrm{~T} 70 \mathrm{~mm}$ no-insulation (RE)Ba $\mathrm{Bu}_{3} \mathrm{O}_{7-\mathrm{x}}$ magnet for an axion haloscope experiment

Review of Scientific Instruments 91, 023314 (2020); https://doi.org/10.1063/1.5124432

Lock-in Amplifiers Find out more today

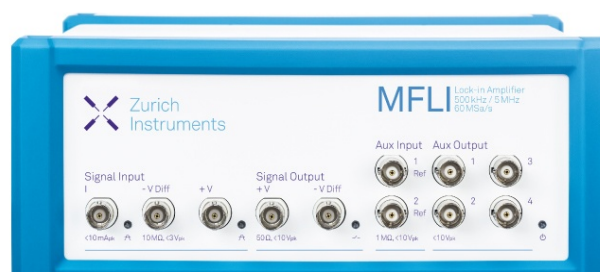

Zurich - Instruments 


\title{
Environmental chamber with controlled temperature and relative humidity for ice crystallization kinetic measurements by atomic force microscopy
}

\author{
Cite as: Rev. Sci. Instrum. 91, 023704 (2020); doi: 10.1063/1.5132537 \\ Submitted: 17 October 2019 - Accepted: 17 January 2020 • \\ Published Online: 6 February 2020
}

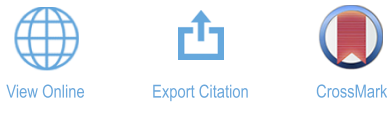

Melisa M. Gianetti, ${ }^{1, a)}$ (D) Julián Gelman Constantin, ${ }^{1,2, b)}$ (D) Horacio R. Corti, ${ }^{1,2}$ (D) and M. Paula Longinotti ${ }^{1, c)}$ (D)

\begin{abstract}
AFFILIATIONS
${ }^{1}$ Instituto de Química Física de los Materiales, Medio Ambiente y Energía (UBA-CONICET), Facultad de Ciencias Exactas y Naturales, Universidad de Buenos Aires, Pabellón II, Ciudad Universitaria, C1428EGA Ciudad Autónoma de Buenos Aires, Argentina

2Departamento de Física de la Materia Condensada, Centro Atómico Constituyentes, Comisión Nacional de Energía Atómica, B1650KNA San Martín, Buenos Aires, Argentina
\end{abstract}

a) Current address: Instituto de Investigaciones en Ciencia y Tecnología de Materiales (INTEMA), Av. Juan B. Justo 4302, B7608FDQ Mar del Plata, Buenos Aires, Argentina.

b) Current address: División de Química Atmosférica, Centro Atómico Constituyentes, Comisión Nacional de Energía Atómica, B1650KNA San Martín, Buenos Aires, Argentina.

c) Author to whom correspondence should be addressed: longinot@qi.fcen.uba.ar

\begin{abstract}
The present work describes the development of an environmental chamber (EC), with temperature and humidity control, for measuring ice growth kinetics over a substrate with an atomic force microscope (AFM). The main component of the EC is an AFM fluid glass cell. The relative humidity (RH) inside the EC is set by the flow of a controlled ratio of dry and humid nitrogen gases. The sample temperature is fixed with an AFM commercial accessory, while the temperature of the nitrogen gas inside the EC is controlled by circulating cold nitrogen vapor through a copper cooler, specially designed for this purpose. With this setup, we could study the growth rate of ice crystallization over a mica substrate by measuring the force exerted between the tip and the sample when they approach each other as a function of time. This experimental development represents a significant improvement with respect to previous experimental determinations of ice growth rates, where $\mathrm{RH}$ and temperature of the air above the sample were determined far away from the ice crystallization regions, in opposition to the present work.
\end{abstract}

Published under license by AIP Publishing. https://doi.org/10.1063/1.5132537

\section{INTRODUCTION}

Ice crystal growth from the vapor phase is an important microphysical process critical for the evolution of atmospheric cold clouds. The rates of water condensation and evaporation on atmospheric particles influence the lifetime properties of clouds, thereby affecting the atmosphere's radiation budget [the balance between incoming energy from the sun and outgoing longwave (thermal) and reflected shortwave energy from Earth], the formation of precipitation, and weather. ${ }^{1,2}$ Quantitative understanding of the kinetics of ice growth is extremely important for atmospheric science, and it is also crucial in many industrial processes, for example, in cryopreservation. A considerable body of literature exists on ice crystallization from the vapor phase. However, most of these studies involve fog chambers and optical detection where the detection limit is in the range from $10^{-6} \mathrm{~m}$ to $10^{-2} \mathrm{~m} .^{3-6}$ Consequently, atomic force microscopy experiments emerged as a higher resolution alternative for this type of experiments since they allow following the crystal growth at the nanoscale level. It should be stressed that for this type of studies, a precise control of the operating conditions, such as 
relative humidity (RH) and temperature, is fundamental, but this is not possible with commercial AFMs. In order to fulfill these requirements, many works were reported in the literature dealing with the development of environmental AFM chambers. ${ }^{7-12}$ For instance, Santore et al. ${ }^{7}$ developed an ad hoc system based on a high vacuum chamber (up to $10^{-6}$ Torr), which allowed them to work under controlled atmospheric conditions to take high quality images of a gold grid and a deposited layer by layer polypyrrole sample. Another interesting design of a controlled RH chamber was developed by Stukalov et al., ${ }^{9}$ which was used to characterize the RH-dependent swelling of small chitosan droplets with diameters between $3 \mu \mathrm{m}$ and $40 \mu \mathrm{m}$. Two decades ago, Zepeda et al. designed a double-jacketed environmental AFM chamber to investigate the surface morphology of ice Ih in its pure phase. In this case, the authors used cold nitrogen and thermoelectric devices for fine temperature regulation with a controlled delivery of water vapor to the substrate to take images of ice surfaces under equilibrium conditions.

In the present work, we describe the development of an environmental AFM chamber for the study of ice growth kinetics over a hard substrate (mica, in this case). Experiments were performed by measuring the force exerted between the AFM tip and the ice sample when they approach each other on a fixed surface location $(x, y)$. AFM force curves over ice are usually used to study the thickness of the quasi-liquid layer (QLL) that exists over a supercooled ice surface and whose properties differ from those of the bulk supercooled liquid at the same temperature. ${ }^{13-17}$ When the tip is away from the ice surface, the force between the tip and the sample is zero. At a certain point, close to the surface, the tip experiences an attractive force, jumping into the ice. In many works, this jump-in distance is interpreted as the QLL thickness although some corrections were proposed by several authors. After this jump-in, a repulsive force is observed because of the penetration/indentation of the tip into the ice sample. When the tip reaches the hard substrate, the force climbs to infinity because of the fact that the substrate cannot be penetrated. In this work, we have determined the ice growth rate by analyzing the indentation distance as a function of time.

Libbrecht ${ }^{18}$ critically reviewed the literature on ice growth measurements, pointing out some sources of systematic errors. Among them is the fact that supersaturation and temperature measurements are usually performed far away from the regions where ice crystallizes. Accordingly, and with an eye toward avoiding this source of error, in this work, we developed an environmental chamber (EC) to control humidity and temperature close to the ice crystallization regions. This work is, to the best of our knowledge, the first time where an environmental AFM chamber was developed for the study of ice growth kinetics.

\section{EXPERIMENTAL DEVELOPMENT}

In this section, we describe the experimental setup developed to study ice growth kinetics.

The environmental AFM has sample temperature control and also temperature and $\mathrm{RH}$ control in the environment above the sample. A commercial AFM by Veeco (currently Bruker), multimode model, using a NanoScope IIIa controller and a Quadrex module, was used. This AFM has a Peltier element for cooling the mica substrate over which ice crystallizes. Sample temperature was controlled with a set of commercial accessories provided by Veeco: the Thermal Applications Controller, the Sample Heater/Cooler Peltier, and the Heater/Cooler Scanner HC-AS-130V. With this setup, we could control the sample temperature between $268.2 \mathrm{~K}$ and $273.2 \mathrm{~K}$ with a precision of $\pm 0.1 \mathrm{~K}$. To control the temperature and $\mathrm{RH}$ in the environment above the sample, an AFM environmental chamber located above the piezoelectric of the AFM was specially designed. Figure 1(a) shows a picture of the environmental chamber located above the mica substrate, and Fig. 1(b) shows a picture of the AFM and environmental chamber under work. The whole setup used in this work is depicted in Fig. 2.

\section{A. AFM environmental chamber (EC)}

The main element of the environmental chamber is an AFM fluid glass cell [6, Fig. 2 and Fig. 1(a)]. The cell has a silicon oring [4, Fig. 2 and the white circle in Fig. 1(a)] to seal the space between the cell and the substrate (3, Fig. 2) over which ice crystallizes, which is located above the Peltier AFM element. It has three built-in ducts, which we used for the inlet (10, Fig. 2), and outlet of gases, and to place a humidity-temperature sensor around $20 \mathrm{~mm}$ from the mica substrate (9, Fig. 2) and to locate a sensor to register
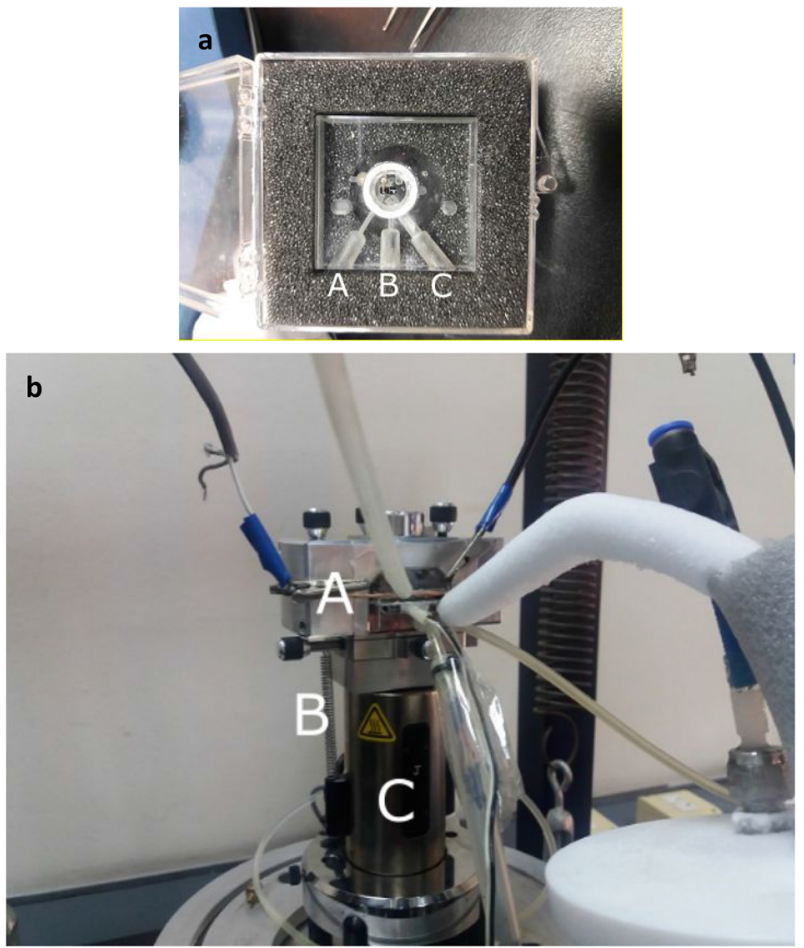

FIG. 1. (a) Fluid glass cell used as the AFM environmental chamber (bottom view) where (A) corresponds to the inlet of gases, (B) corresponds to the outlet of gases and the temperature and $\mathrm{RH}$ humidity sensor, and $(\mathrm{C})$ corresponds to the glass cell temperature sensor and (b) AFM and environmental chamber under work with the fluid glass cell (A) fixed on the piezoelectric element (C) by using springs (B); the tube which is covered with condensed ice corresponds to the inlet of gases used to control the temperature of the nitrogen gas above the sample (item 7 in Fig. 2). 


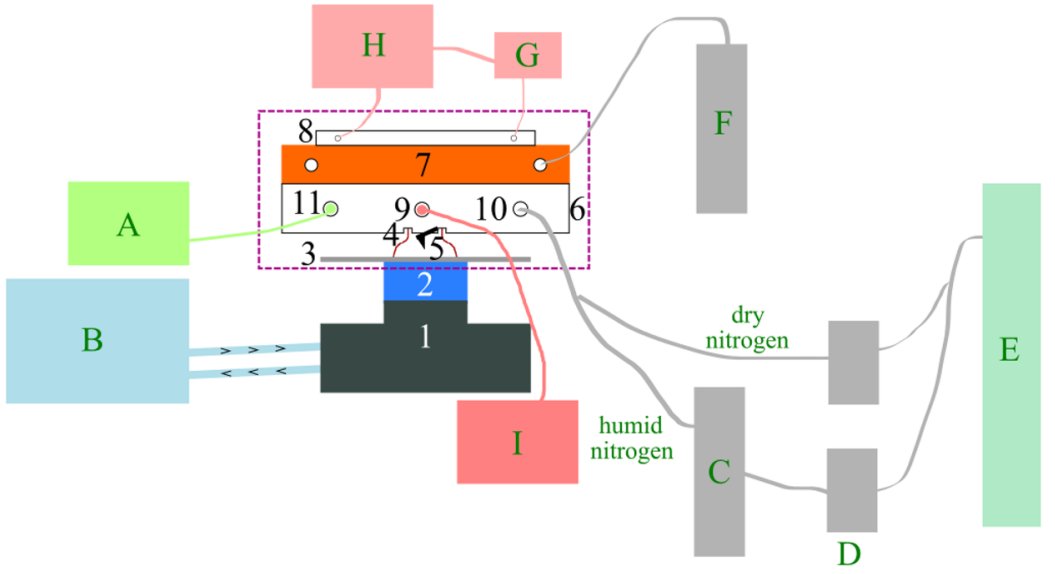

FIG. 2. Schematic diagram of the experimental setup showing the (1) AFM piezoelectric, (2) Peltier element, (3) mica substrate located in the EC (dashed square), (4) silicone o-ring, (5) AFM tip, (6) fluid glass cell, (7) copper cooler, (8) heating resistance, ( $(1-9)$ gas outlet and humidity/temperature sensor, (10) gas inlet, and (11) glass cell temperature sensor; the system for controlling the temperature and $\mathrm{RH}$ of the gaseous nitrogen above the sample includes a humidity-temperature sensor [A, located in hole (11)], a cooling bath which circulates water inside the piezoelectric to thermostatize the high temperature side of the Peltier element (B), a bubbler containing liquid water (C), two Alicat flow controllers (D), a nitrogen cylinder $(E)$, a dewar with liquid nitrogen $(\mathrm{F})$, a PID temperature controller $(\mathrm{G})$, and a voltage source $(\mathrm{H})$ which controls the temperature according to that measured by a temperature sensor located in the copper cooler. the temperature of the glass cell, $8 \mathrm{~mm}$ away from the mica substrate (11, Fig. 2). In order to control the RH inside the chamber, humid and dry gaseous nitrogen are mixed at a controlled ratio using two flow controllers (D, Fig. 2), with maximum flow rates of $200 \mathrm{~cm}^{3} \mathrm{~min}^{-1}$ and $50 \mathrm{~cm}^{3} \mathrm{~min}^{-1}$, for dry and humid nitrogen, respectively. To control the temperature of the gases in contact with the sample, we designed a copper cooler (7, Fig. 2 and the picture shown in Fig. 3) based on the cold flowing nitrogen vapor through the channels of a copper block. The cold nitrogen vapor is generated by the evaporation of liquid nitrogen, when it comes in contact with the copper channel walls, which flows by the siphon effect from a dewar (F, Fig. 2). The nitrogen flow rate is adjusted with a vent valve in the dewar flask that controls the overpressure. Fine temperature control is achieved with an in-house developed heater located on top of the copper cooler. The heater is made with nichrome (a nickel-chromium alloy) wire coiled around a mica sheet; it is electrically isolated with an additional mica sheet on the bottom side and a glass slide on the top side. The total heater resistance is $18 \Omega$.

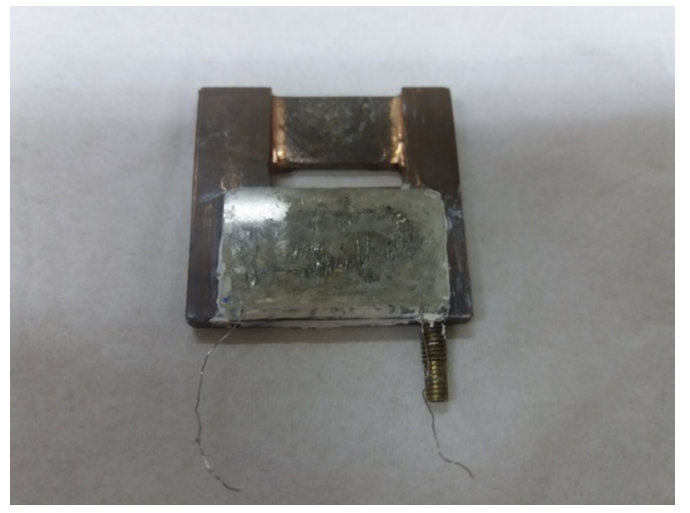

FIG. 3. Copper cooler designed to control the temperature of the nitrogen gas located above the sample. The picture shows a copper tube for the inlet of cold nitrogen vapor and two nichrome wires which are coiled around a mica sheet for heating the copper cooler. The terminals of the nichrome wires are connected to the PID Controller (G, Fig. 2) by crocodile clips, which can be observed in Fig. 1(b).
The heater is powered by a PID controller (TERMOLD, NG-2 model), which measures the temperature of the copper cooler with a platinum resistance sensor (Honeywell HEL-777-A-T-0, 100). This system allows controlling the temperature of the copper cooler with fluctuations below $\pm 0.5^{\circ} \mathrm{C}$.

\section{B. Force curve measurements}

The force exerted between the AFM tip and an ice thin film crystallized over a hard substrate when they approach each other can give information of the ice film thickness. The force curve for the interaction of the AFM tip and an ice film crystallized over a hard substrate looks similar to the scheme given in Fig. 4, where three regions can be clearly distinguished:

1. Non-contact region (where the force is zero).

2. Ice penetration/indentation region (the positive slope): repulsive forces arise when the tip penetrates the ice film. The displacement of the tip in this region is related to the ice layer thickness (the ice indentation distance).

3. The tip reaches a hard substrate over which ice is deposited (mica), and hence, repulsive forces tend to climb to infinity (infinity slope).

Some force curves, for the interaction of the AFM tip and ice, show a jump-in (a negative slope between regions 1 and 2) because an attractive force associated with the QLL is observed over the solid ice film. In this figure, no jump-in region is depicted since no jump-in was detected with the tips used in this work. The width of region 2 represents the thickness of the ice layer.

Force curves were measured as a function of time when ice crystallizes from the vapor phase over the mica substrate. The analysis of the indentation distance (region 2 in Fig. 4) as a function of time can give information of the ice growth rate.

Force curves were registered at a frequency of $1.744 \mathrm{~Hz}$ and sampling 8192 data points per curve. Measurements were performed with an AFM tip (a Pt/Ir-coated tip) provided by Nanosensors, whose characteristics are summarized in Table I. The characteristics of the tip (shape and chemistry) may affect the QLL thickness determinations, ${ }^{17,19}$ but none of these factors would significantly affect the ice thickness measurements used to determine the ice 


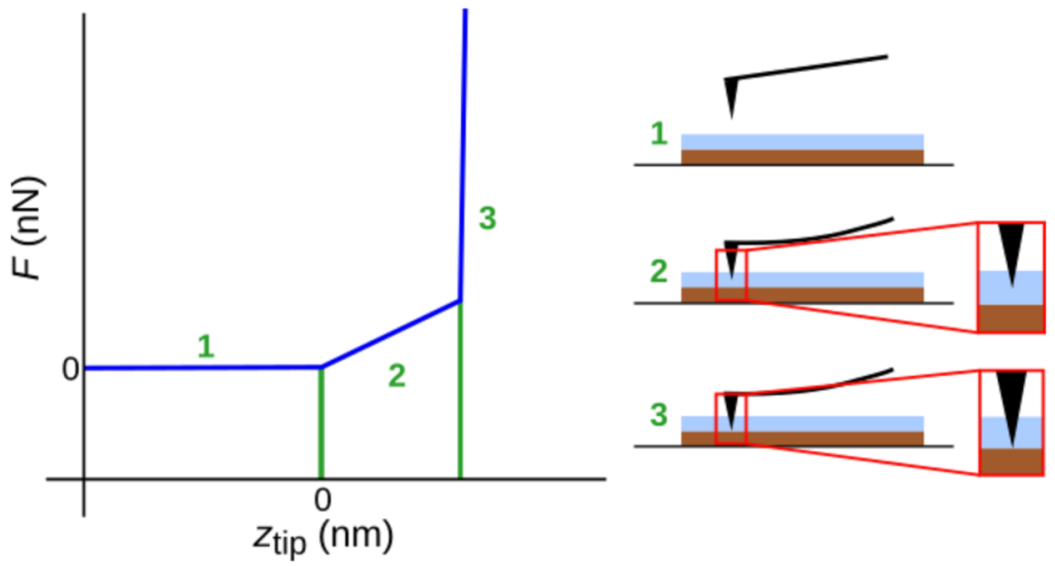

FIG. 4. Schematic diagram of the shape of AFM force curves (left panel) for the interaction of an AFM tip (black) with ice (light blue) deposited over a mica substrate (brown) Different characteristic zones correspond to the (1) noncontact region, (2) indentation region, and (3) contact with the mica substrate. The attractive force between the tip and the QLL is not shown because in the experiments performed in this work, this attractive force was not observed due to the high force constant of the tip employed. The right-hand panel depicts the characteristic zones shown in the force curve. The QLL is not shown in the right-hand panel since its thickness is negligible in relation to the ice thickness.

growth rates. Raw data generated by the AFM operating software (NanoScope 5.30r3, Veeco) were exported with NanoScope Analysis 1.40 (Bruker) and post-processed with Microsoft Excel 2010.

The raw voltage vs piezoelectric displacement $\left(z_{\text {piezo }}\right)$ data are transformed into force plots (force vs indentation depth, $z_{\text {tip }}$ ). Quantitative analysis of force curves requires several calibrations, which are detailed in a previous study ${ }^{17}$ and summarized in Sec. II C.

In the experiments detailed in this work, we normalized the curves by fixing $z_{\text {tip }}=0$ at the $z$ position where the tip starts to indent the ice. The definition of $z_{\text {tip }}=0$ for samples that do not behave as infinitely hard substrates is not straightforward ${ }^{20}$ and can affect some indirect AFM measurements (such as the study of viscoelastic or plastic deformation of samples). However, here, we performed direct measurements, and the definition of $z_{\text {tip }}=0$ does not affect the ice indentation distances used for the calculation of ice growth rates.

\section{AFM calibration details}

As mentioned, quantitative analysis of force curves requires several calibrations. The piezoelectric was periodically calibrated in $x, y$, and $z$ directions using Nanoscope 5.30r3 and a commercial calibration grid (Veeco VGRP-15M, platinum covered silicon, with

TABLE I. Characteristics of the AFM tip used for the force curve measurements.

\begin{tabular}{lc}
\hline \multicolumn{1}{c}{ Material } & Silicon \\
\hline Tip coating & Pt/Ir \\
Manufacturer & Nanosensors \\
Model & PPP-EFM \\
Tip radius (nominal) & Not specified \\
(maximum) & $25 \mathrm{~nm}$ \\
Spring constant $\left(\mathrm{N} \mathrm{m}^{-1}\right)$ (average) & 2.8 \\
(minimum) & 0.5 \\
(maximum) & 9.5 \\
Resonance frequency $(\mathrm{kHz})$ (average) & 75 \\
(minimum) & 45 \\
(maximum) & 115 \\
\hline \hline
\end{tabular}

square pits of $10 \mu \mathrm{m} \times 10 \mu \mathrm{m}$ and $180 \mathrm{~nm}$ depth). This assures accuracy on the $z_{\text {piezo }}$ coordinate.

In order to obtain force curves in the format given in Fig. 4, several calibrations should be performed. First, raw deflection data (informed as potential between $-9.68 \mathrm{~V}$ and $+9.68 \mathrm{~V}$ ) is converted to deflection distance. The way to do that, is measuring force curves on an "infinitely hard" substrate (mica, in this case). Then, we analyzed those curves under the assumption that in the contact region, there is no indentation of the sample. ${ }^{20}$ Thus, the true distance between the tip and the sample remains constant: the piezoelectric displacement is compensated by the cantilever deflection. Hence, the slope of this region of the force curve can be used to calibrate the ratio between the potential measured on the detector and the cantilever deflection in distance units. This slope is usually called deflection sensitivity (Sens) and depends mainly on the cantilever geometry and the laser alignment on the cantilever (and other parameters of the optical path of the beam) and secondarily on temperature gradients. We used the deflection sensitivity measured at the appropriate temperature to convert the raw deflection as follows:

$$
\operatorname{Defl} / \mathrm{nm}=\frac{\mathrm{Defl} / \mathrm{V}}{\operatorname{Sens}(\mathrm{V} / \mathrm{nm})},
$$

where Defl is the cantilever deflection (in $\mathrm{nm}$ and V). We determined Sens for each experiment over bare mica (before ice deposition) at the same temperature used later to study ice growth.

The conversion of the cantilever deflection to force $(F)$ assumes a simple Hooke-like law ( $F=-K$.Defl, where $K$ is the spring constant of the cantilever). Calibration of the tip spring constant, $K$, used to convert the measured deflection into force, was not necessary in this work since the ice growth rate does not depend on it. Thus, the value for the spring constant used in these experiments is the one reported by the manufacturer and included in Table I. Moreover, it should be stressed that for the small temperature range studied in this work, it can be considered that $K$ has the same value for all the experiments.

Force curves are usually presented as a function of $z_{\text {tip }}$ (the tip to sample distance) in the abscissa axis, while measurements are performed as a function of $\mathrm{z}_{\text {piezo }}$. The conversion equation is simple,

$$
z_{\text {tip }} / \mathrm{nm}=z_{\text {piezo }} / \mathrm{nm}-\operatorname{Defl} / \mathrm{nm}=z_{\text {piezo }} / \mathrm{nm}-\frac{\text { Defl } / \mathrm{V}}{\operatorname{Sens} /(V / n m)} .
$$


TABLE II. Operational parameters for the different experiments performed with the environmental chamber.

\begin{tabular}{lcccc}
\hline \hline Experiment & $T(\mathrm{~K})$ & $\Delta T(\mathrm{~K})$ & $\mathrm{RH}(\%)( \pm 13 \%)$ & $T_{\mathrm{N} 2}(\mathrm{~K})$ \\
\hline 1 & 268.2 & $5.0 \pm 0.1$ & 144 & $276.2 \pm 0.5$ \\
2 & 269.7 & $3.5 \pm 0.1$ & 114 & $274.2 \pm 0.5$ \\
3 & 268.2 & $5.0 \pm 0.1$ & 120 & $276.2 \pm 0.5$ \\
4 & 268.2 & $5.0 \pm 0.1$ & 120 & $276.2 \pm 0.5$ \\
\hline \hline
\end{tabular}

\section{Ice crystallization procedure}

Ice growth kinetic measurements were performed when ice crystallized from the vapor phase over a flat mica surface. A $12 \mathrm{~mm}$ diameter mica disk (supplied by Veeco) was cleaved with an adhesive tape before every experiment to produce a fresh and flat surface. The disk was then placed on the Peltier element and fixed with silicone thermal paste (Thermal Joint Compound, Type 120 Silicone, Wakefield Solutions) for effective heat transfer. The cantilever holder was placed above the mica disk sealing the EC, as shown in Fig. 2. Dry nitrogen was circulated inside the chamber to dry up the environment, and the Peltier's temperature was set to $273.2 \mathrm{~K}$. Later, dry nitrogen was slowly mixed with a flux of nitrogen gas saturated with water vapor (Fig. 2). The resulting $\mathrm{RH}$ was set below the ice saturation value at $273.2 \mathrm{~K}$ in such a way that ice crystallization was not observed, but it could occur below this temperature. Once the RH was constant, force curves were continuously registered (measuring 34.4 curves/min), while the temperature was slowly reduced until a pre-determined constant value was attained. When this final constant temperature value was reached and ice deposition was detected (obtaining force curves with a shape similar to the example curve given in Fig. 4), the time was set equal to zero $(t=0)$. The time evolution of ice thickness over the surface was determined. Force curves obtained before ice deposition were used for calibrating the

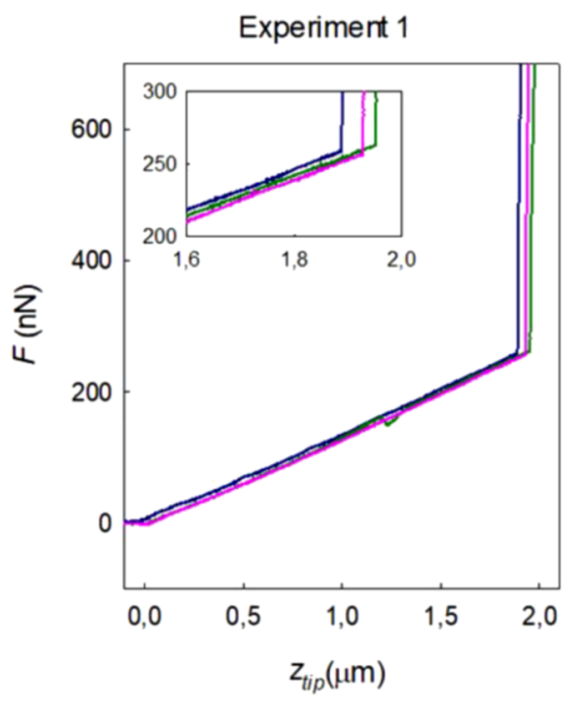

Experiment 3

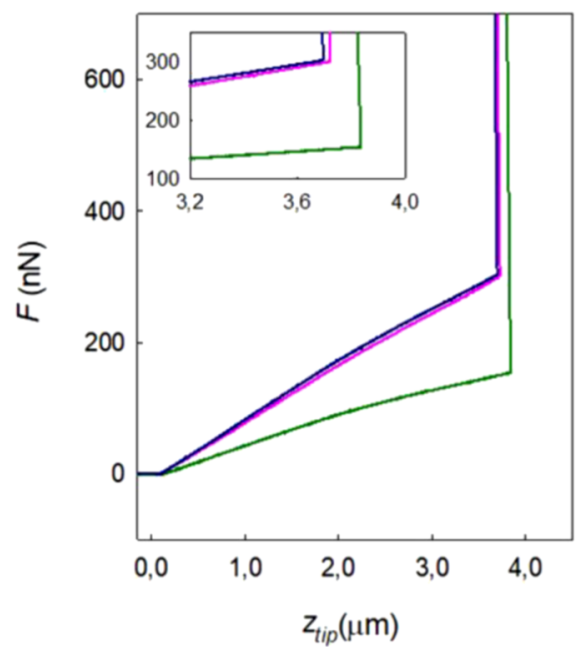

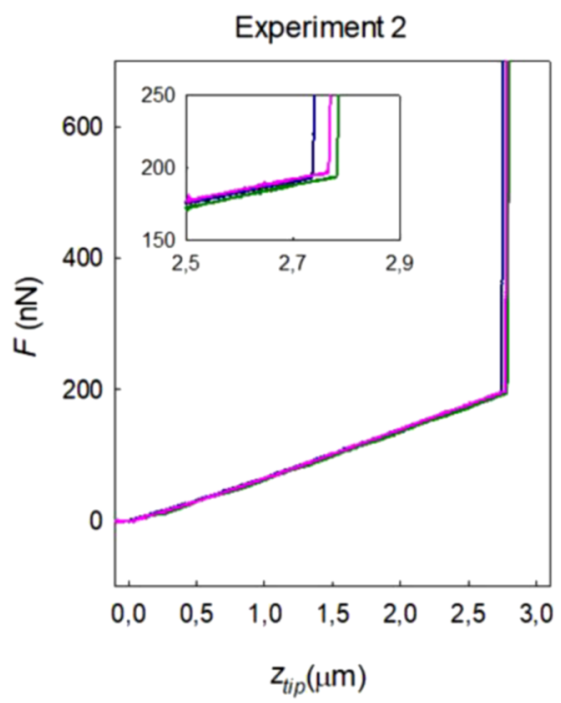

Experiment 4

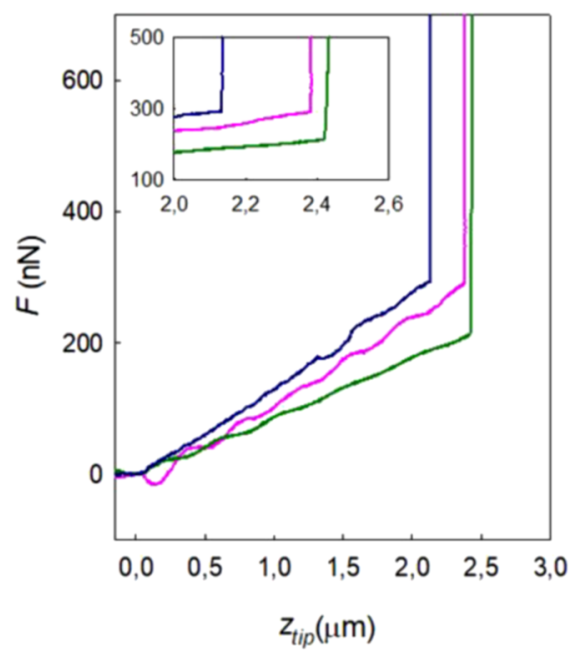

FIG. 5. Representative force curves for experiments $1-4$. Insets show ice growth details. Experiment 1: at 0 min (blue), 0.8 $\mathrm{min}$ (pink), and $1.7 \mathrm{~min}$ (green). Experiment 2: at $15.4 \mathrm{~min}$ (blue), $16.0 \mathrm{~min}$ (pink), and $17.7 \mathrm{~min}$ (green). Experiment 3: at $6.4 \mathrm{~min}$ (blue), $8.4 \mathrm{~min}$ (pink), and $10.4 \mathrm{~min}$ (green). Experiment 4: at 2.0 min (blue), $10.1 \mathrm{~min}$ (pink), and $19.4 \mathrm{~min}$ (green). 
deflection sensitivity, that is, to transform the raw data in force curves as in the form of Fig. 4 (as explained in Sec. II C). In practice, the precise moment when ice crystallization began could not be detected because the photodiode detector should be repositioned during the measurement in order to register complete force curves. Due to this instrumental limitation, in these experiments, we were not able to study the initial stages of ice crystallization. That is why this experimental setup is developed for growth rate measurements of ice growing on itself, without influence of the substrate over which it crystallizes.

\section{RESULTS AND DISCUSSION}

In this work, we performed ice growth rate determinations in a temperature range between $268.2 \mathrm{~K}$ and $270.7 \mathrm{~K}$, that is, to say, supercooling $\left(\Delta T=T_{f}-T\right)$ between $5.0 \mathrm{~K}$ and $2.5 \mathrm{~K}$. Ice deposition was observed for a relative humidity $(\mathrm{RH})$ of around $120 \% \pm 13 \%$ (saturation with respect to ice) for all the experiments. The reported uncertainty in the RH determination takes into account the nominal precision of the humidity sensor and the conversion from $\mathrm{RH}$ measured outside the cell to saturation with respect to the ice sample. The humidity uncertainty is quite large, as expected, considering that we are measuring very low vapor pressures at the analyzed temperature range. However, some results obtained in a previous study $^{17}$ showed that during ice deposition at constant temperature, $\mathrm{RH}$ attained a constant value of $(102.5 \pm 4.4) \%$, indicating that probably the uncertainty in the $\mathrm{RH}$ is overestimated. Table II summarizes the sample temperature, supercooling, and temperature and $\mathrm{RH}$ of the humid nitrogen above the sample for the experiments performed. It should be noted that experiments at supercooling above $5 \mathrm{~K}$ are difficult to achieve due to difficulties in attaining stable sample temperatures at larger supercooling.

Figure 5 shows representative force curves obtained during crystallization of ice in experiments 1-4.

These curves show an increment in the indentation depth $\left(z_{\text {tip }}\right.$ distance in region 2 of Fig. 5) with increasing time, showing ice growths over the mica surface. From this figure, it can be noticed that the indentation slope is not constant for a given experiment. This can be explained considering that ice is growing over the mica sample, with subtle non-systematic changes in the temperature of the sample and temperature and relative humidity of the nitrogen gas above the sample with time. Moreover, after repeated indentation, the pressure of the tip can compact the growing ice layer, which can be another reason for the change in the indentation slope with time. In addition, it can be observed in experiment 4 that the ice indentation region is not linear, which can be related to the existence of microscopic heterogeneities in the tip, as pointed out by Pittenger $e t$ al. ${ }^{14}$ The slope of the indentation curve depends on the tip velocity and can also give information related to the deformation of ice during indentation (plastic deformation vs viscoelastic behavior of the QLL between the tip and the ice sample ${ }^{14,21}$ ). However, this analysis is out of the scope of this work.

The growth rates can be calculated from the slopes of the time evolution curves of the indentation distance. These indentation distances, called $d$, are plotted in Fig. 6 as a function of time for four experiments. The error in $d$ is $\pm 1 \mathrm{~nm}$ because of the noise in the deflection signal.
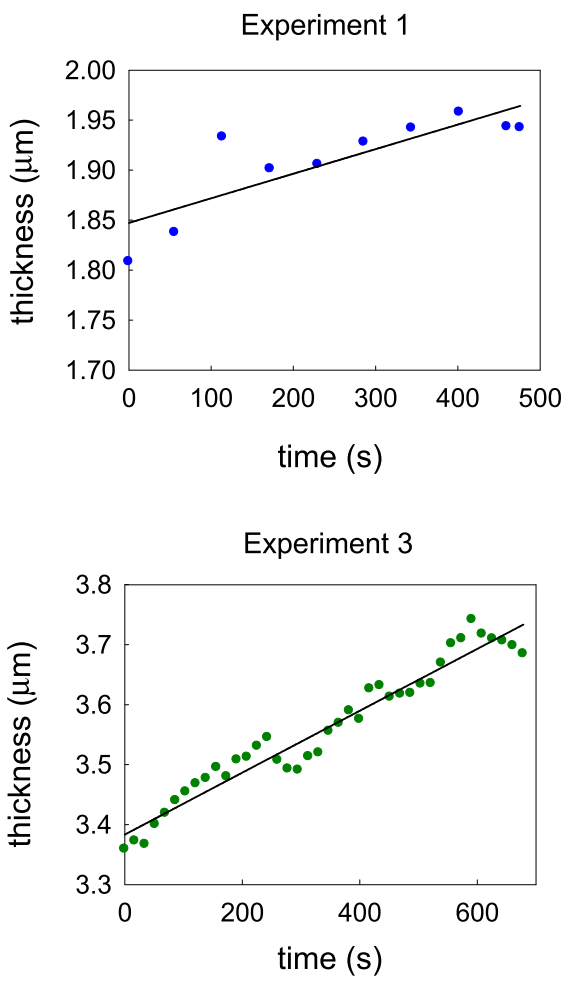

Experiment 2

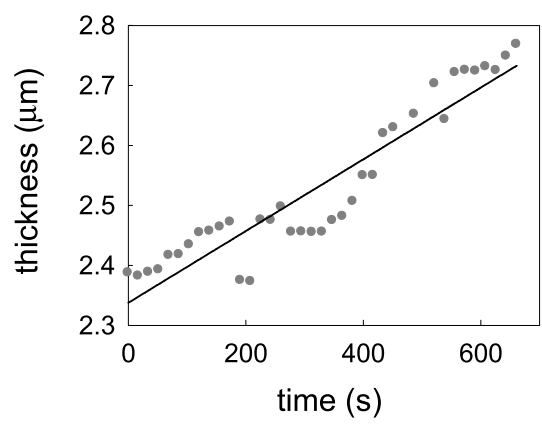

Experiment 4

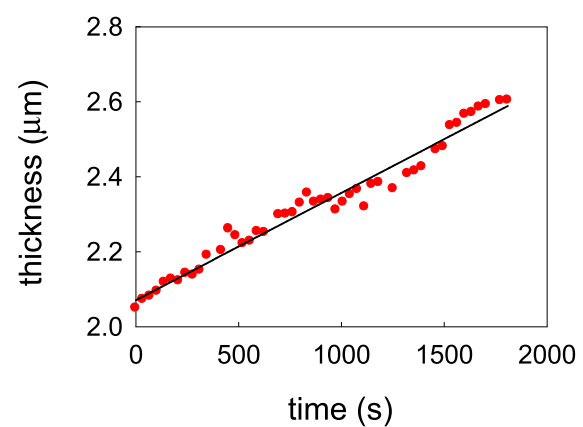

FIG. 6. Time evolution of the ice thickness (d). Linear fits (black lines), used to calculate ice growth rates, are shown in all figures. 
TABLE III: Ice growth rates obtained for experiments 1-4.

\begin{tabular}{cc}
\hline \hline Experiment & $v\left(\mathrm{~nm} \mathrm{~min}^{-1}\right)$ \\
\hline 1 & $15 \pm 3$ \\
2 & $36 \pm 2$ \\
3 & $31 \pm 1$ \\
4 & $17.2 \pm 0.4$ \\
\hline
\end{tabular}

Table III summarizes the ice growth rates obtained for experiments $1-4$, determined by the slopes of the linear regressions plotted in Fig. 6.

Considering the dispersion of the data reported in Table III, an approximate value for the ice growth rate can be calculated, $v=25 \pm 10 \mathrm{~nm} \mathrm{~min}{ }^{-1}$, in the temperature and $\mathrm{RH}$ range analyzed. The dispersion of the data can be explained considering that different crystallization regions were analyzed in all experiments, that is, different microscopic regions of the sample were studied. The ice preparation procedure described produces polycrystalline ice, as it is likely to be found in the environment. However, the ice growth rate is expected to depend on the exposed crystal face, which can neither be identified nor selected with this procedure. This experimental technique could be complemented with the preparation of ice single crystals, which could later be introduced in the fluid cell to study the ice growth rate under more controlled conditions.

Our result is of considerable significance since it is the first reported ice growth rate value obtained with an environmental AFM, in which the temperature and $\mathrm{RH}$ close to the ice crystallization regions were controlled. Future studies using this setup could help to reveal the complex dependence, with temperature and $\mathrm{RH}$, of the ice growth mechanism.

\section{CONCLUSIONS}

In this work, we show the development of an environmental chamber for ice growth measurements in the nanoscale level with an AFM at controlled temperature and RH. The kinetics of ice growth were determined by analyzing the force curves exerted between the AFM tip and the ice sample as they approach each other as a function of time at a fixed $(x, y)$ position. The technique developed in the present work has an additional advantage with respect to previously reported results of ice growth kinetics, considering the fact that temperature and $\mathrm{RH}$ could be controlled and measured close to ice crystallization regions. Even though the supercooling and $\mathrm{RH}$ ranges studied are rather limited, this work proves the usefulness of environmental AFM for studying ice growth kinetics.

\section{ACKNOWLEDGMENTS}

The authors thank financial support from ANPCyT (Grant No. PICT 2013-2238). M.P.L., J.G.C., and H.R.C. are members of CONICET. M.M.G. and J.G.C. thank Ph.D. fellowships from CONICET.

\section{REFERENCES}

${ }^{1}$ T. Bartels-Rauch, V. Bergeron, J. H. E. Cartwright, R. Escribano, J. L. Finney, H. Grothe, P. J. Gutiérrez, J. Haapala, W. F. Kuhs, J. B. C. Pettersson, S. D. Price, I. Sainz-Díaz, D. J. Stokes, G. Strazzulla, E. S. Thomson, H. Trinks, and N. Uras-Aytemiz, Rev. Mod. Phys. 84, 885 (2012).

${ }^{2}$ C. Hoose, J. E. Kristjánsson, J. P. Chen, and A. A. Hazra, J. Atmos. Sci. 67, 2483 (2010).

${ }^{3}$ M. L. Eastwood, S. Cremel, C. Gehrke, E. Girard, and A. K. Bertram, J. Geophys. Res.: Atmos. 113, D22203, https://doi.org/10.1029/2008jd010639 (2008).

${ }^{4}$ T. Zolles, J. Burkart, T. Häusler, B. Pummer, R. Hitzenberger, and H. Grothe, J. Phys. Chem. A 119, 2692 (2015).

${ }^{5}$ J. Hallett, J. Atmos. Sci. 22, 64 (1965).

${ }^{6}$ D. Lamb and P. V. Hobbs, J. Atmos. Sci. 28, 1506 (1971).

${ }^{7}$ M. Sartore, R. Pace, P. Faraci, D. Nardelli, M. Adami, M. K. Ram, and C. Nicolini, Rev. Sci. Inst. 71, 2409 (2000).

${ }^{8}$ S. Zepeda, Y. Yeh, and C. A. Orme, Rev. Sci. Inst. 72, 4159 (2001).

${ }^{9}$ O. Stukalov, C. A. Murray, A. Jacina, and K. R. Dutcher, Rev. Sci. Inst. 77, 033704 (2006).

${ }^{10}$ J. Lievonen, K. Ranttila, and M. Ahlskog, Rev. Sci. Inst. 78, 043703 (2007).

${ }^{11}$ S. S. Nonnenmann and D. A. Bonnell, Rev. Sci. Inst. 84, 073707 (2013).

${ }^{12}$ I. Gaponenko, L. Gamperle, K. Herberg, S. C. Muller, and P. Paruch, Rev. Sci. Inst. 87, 063709 (2016).

${ }^{13}$ A. Döppenschmidt and H. J. Butt, Langmuir 16, 6709 (2000).

${ }^{14}$ B. Pittenger, S. C. Fain, Jr., M. J. Cochran, J. M. K. Donev, B. E. Robertson, A. Szuchmacher, and R. M. Overney, Phys. Rev. B 63, 134102 (2001).

${ }^{15}$ B. Pittenger, D. J. Cook, C. R. Slaughterbeck, and S. C. Fain, Jr, J. Vac. Sci. Technol., A 16, 1832 (1998).

${ }^{16}$ A. Döppenschmidt, M. Kappl, and H. J. Butt, J. Phys. Chem. B 102, 7813 (1998).

${ }^{17}$ J. Gelman Constantin, M. M. Gianetti, M. P. Longinotti, and H. R. Corti, Atmos. Chem. Phys. 18, 14965 (2018).

${ }^{18}$ K. G. Libbrecht, arXiv:cond-mat/0411662, archive paper (2004).

${ }^{19}$ J. Gelman Constantin, M. A. Carignano, H. R. Corti, and I. Szleifer, J. Phys. Chem. C 119, 27118 (2015).

${ }^{20}$ H. J. Butt, B. Cappella, and M. Kappl, Surf. Sci. Rep. 59, 1 (2005).

${ }^{21}$ H. J. Butt, A. Döppenschmidt, G. Hüttl, E. Müller, and O. I. Cinogradova, J. Chem. Phys. 113, 1194 (2000).

${ }^{22}$ X. Kong, P. Papagiannakopoulos, E. S. Thomson, N. Markovic, and J. B. C. Pettersson, J. Phys. Chem. A 118, 3973 (2014). 